\title{
An asymmetry in transmodal perceptual learning
}

\author{
BARRY HUGHES and WILLIAM EPSTEIN \\ University of Wisconsin, Madison, Wisconsin \\ SANDRA SCHNEIDER \\ University of South Florida, Tampa, Florida \\ and \\ ALISON DUDOCK \\ University of Wisconsin, Madison, Wisconsin
}

\begin{abstract}
Transmodal perceptual learning was examined in a canonical same/different paradigm. Subjects naive to vibrotactile stimulation and unfamiliar with the task were asked to discriminate sequentially presented shapes moving laterally across an aperture. On all trials, the shapes were presented either visually and then vibrotactually or in the opposite modality order, and on all trials the shapes moved in opposite directions. Analyses of the data revealed that although perceptual learning was evident in both groups, the rate of the learning was more rapid in the visual-vibrotactile group. This interaction of modality order and practice was significant and was considered in terms of $\mathbf{E}$. J. Gibson's theory of perceptual learning and in terms of the constructs, suggested by J. J. Gibson, of available and accessible information-in-stimulation and the education of attention.
\end{abstract}

The present experiment extends our investigation of perceptual learning in the discrimination of patterns delivered via vibrotactile stimulation. By deploying vibrotactile stimulation, we intend to allow greater scope for learning than would be present in a task that involved a regularly utilized modality. In our earlier experiments (Epstein, Hughes, Schneider, \& Bach-y-Rita, 1989), subjects performed an identification task that required them to select from an array of static visual shapes a match for a dynamic, laterally moving vibrotactile shape. The vibrotactile shape was delivered to the fingerpad in a manner resembling the procedure used in visual anorthoscopic presentations (e.g., Anstis \& Atkinson, 1967; Casco \& Morgan, 1984; Morgan, Findlay, \& Watt, 1982; Rock, 1981). The following were the principal results: (1) Most subjects showed significant improvement with practice; (2) robust transfer from a "training" set of shapes to a set never before encountered was observed; and (3) although there was sizable interindividual variability in level of identification accuracy, there was a striking similarity among all subjects with respect to the perceptual confusability of certain shapes.

This research has been supported by Grant 1 R01 EY0 6719 from the National Institutes of Health, awarded to William Epstein. We gratefully acknowledge the computer programming of Art Walasek, and the contributions of Jack Loomis and an anonymous reviewer. Correspondence should be directed to William Epstein, Department of Psychology, University of Wisconsin-Madison, 1202 W. Johnson Street, Madison, WI 53706.
Our interpretation of these results drew heavily on the perceptual learning constructs promoted by $E$. J. and J. J. Gibson (E. J. Gibson, 1969; E. J. Gibson \& J. J. Gibson, 1955; J. J. Gibson, 1966, 1979). We supposed that learning was a process of differentiating the relational variables that are contained in dynamic patterns and that underlie those patterns' identification. Differentiation also supports transfer when the training and test sets are articulated by similar relational variables. Finally, the evidence of intermodal pattern matching is consistent with the concept of amodal information, which we derived from J. J. Gibson's concept of perceptual equivalence. Epstein (1985, p. 422) suggested that information in stimulation could be considered amodal if two conditions are satisfied-if (1) the information is carried by spatiotemporal patterns of stimulation that exhibit the same form, and if (2) the information affords different perceptual systems with equivalent descriptions of environmental states or events.

In the present experiment, we had three objectives: (1) We wished to extend the generality of our findings of perceptual learning to a canonical same/different task. (2) Inasmuch as our emphasis had been on the pickup of spatiotemporal vibrotactile structures (relational variables), we wished to deliver the visual comparators in the dynamic mode; in this way, isomorphic vibrotactile and optical spatiotemporal structures would be made available. (3) In the successive same/different task with the deployment of different modalities, the effect on learning of the order of modality could be examined. To our knowledge, such effects have not otherwise been studied in a learning 
paradigm. (Reviews of cross-modal matching are provided by Jones [1981], by Marks [1978], and most recently by Garbin [1988].) An interaction of order of modality and practice would have implications for the model of perceptual learning and the construct of amodal information.

\section{METHOD}

\section{Apparatus}

An Optacon (Telesensory Systems, Inc.), containing a 6 column $\times$ 24 row tactile array whose tactors vibrate at $230 \mathrm{~Hz}$ when activated, was used to present dynamic vibrotactile patterns to the left index fingerpad of subjects. This arrangement has been described elsewhere (e.g., Epstein et al., 1989; Schneider, Hughes, Epstein, \& Bach-y-Rita, 1986); the only changes for the present experiment were the interface with a different laboratory microcomputer (Telex 1280) and the utilization of the Optacon's analog visual LED display, which was located on a table approximately $90 \mathrm{~cm}$ in front of the subjects. At this distance, the LED array of the visual monitor subtended approximately $8.0^{\circ} \times 4.7^{\circ}$ of visual angle. The subjects viewed the display binocularly, and no major constraint was placed on head or eye movements. The subjects wore a soundattenuating headset (ERB Model 211C) and used the computer keypad to register their responses. The intensity setting of the Optacon was set by each subject within a defined range so as to be comfortable without detracting from the subjective clarity of the stimulus events.

\section{Stimulus Events}

Five shapes, shown in Figure 1, measured 18 rows $\times 16$ columns and closely resembled those used by Epstein et al. (1989). They
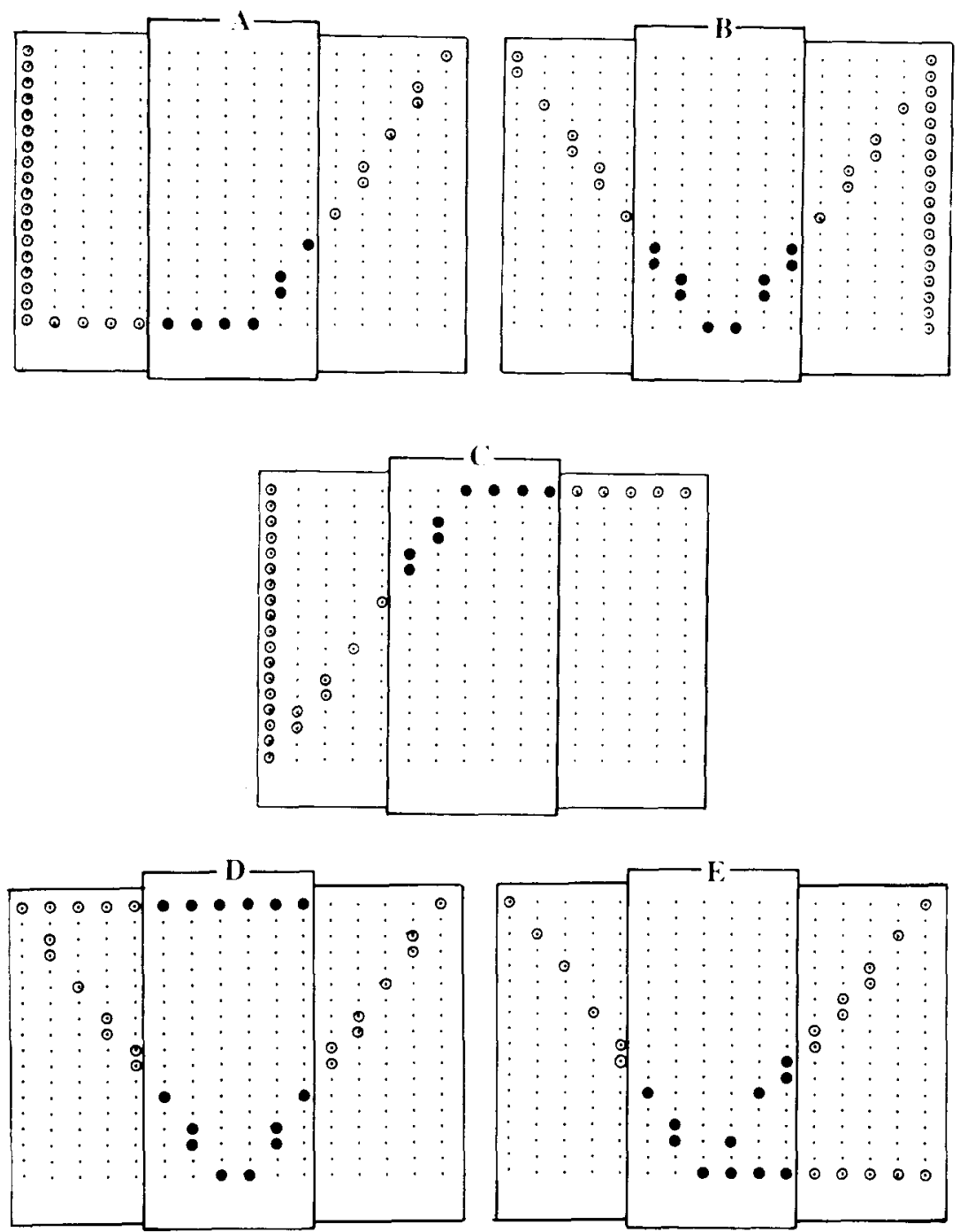

Figure 1. A schematic representation of the five patterns used in the experiment. The smallest dots represent the spatial configuration of tactors or LEDs used in the construction and presentation of the shapes. The inner frame represents the array across which the patterns moved and through which they were perceived. The circles surrounding the dots represent the pattern shapes; the filled circle surrounds correspond to the activated tactors/LEDs during event presentation. The patterns were shifted column by column to either the left or the right, and the representation shown represents this sequence at one instant in time. 
were constructed by joining three lines in different arrangements, and they lacked symmetry, closedness, and obvious meaning. The patterns' lateral motion (i.e., along the left-right dimension) was simulated by presenting distinct frames for a fixed duration of 20 vibratory cycles (i.e., $87 \mathrm{msec}$ per frame), and shifting the frame left or right with a 0 -msec interframe interval. This resulted in a simulated constant lateral velocity of 11.5 columns $/ \mathrm{sec}$, corresponding to approximately $10.3 \mathrm{~cm} / \mathrm{sec}$ on the skin and $8.1^{\circ}$ of lateral visual angle per second. Irrespective of modality or direction of motion, each entire event was of equal duration $(1.827 \mathrm{sec})$.

On a given trial, two patterns were sequentially presented. The pair of patterns on each trial comprised either two identical patterns (e.g., A-A) or two different patterns (e.g., A-C). Both same and different pairs differed in two respects: (1) One pattern was always delivered to the (vibro)tactile perceptual system, whereas the other pattern was always delivered to the visual system (with order an independent variable); and (2) the direction of motion of the two patterns always differed (but was counterbalanced across blocks). A 400-msec interval separated the conclusion of one event and the onset of the second.

\section{Design}

The experiment consisted of a mixed design with repeated measures. Random counterbalancing was used to assign subjects to the two groups, corresponding to the order (vibrotactile-visual or visual-vibrotactile) with which the patterns were presented. Each subject completed eight blocks of trials over 2 separate days. A block of trials comprised 40 trials: 20 same pairs and 20 different pairs. The same pairs were four repetitions of each of the five patterns, and the different pairs included one repetition of each offdiagonal pair (a method used to equate the probabilities of same and different pairs occurring within a block). The dependent measure was the correctness of the same/different judgment.

\section{Procedure}

Subjects were told before the experiment that the task required them to make accurate discriminations of moving shapes. They were informed that the same and different pairs would occur equally often and that the two patterns in each pair would move in opposite directions. They were instructed to defer responding until presentation of both patterns was concluded, but not to delay the response for more than $5 \mathrm{sec}$ after completion of the second event. No feedback or knowledge about the results was provided.

Inasmuch as the Optacon's tactile array and visual monitor are rigidly configured in parallel, it was not possible to have the computer send an event to either display without its necessarily going to the other. Consequently, the experimenter instructed the subjects in the visual-vibrotactile group to remove the finger from the array and visually observe the first moving shape, and then to place the left index fingerpad on the array in preparation for the second event in the trial. The reverse order applied to the vibrotactile-visual group. The experimenter covered or uncovered the entire visual monitor in accordance with the experimental condition and was in a position to determine that her verbal reminders to remove the finger from the array were conformed with on all trials.

\section{Subjects}

The subjects were 9 male and 7 female undergraduate students at the University of Wisconsin who participated for course credit or cash payment (at $\$ 4$ per hour). No subject had previously used either of the particular arrays.

\section{RESULTS}

The data were subjected to several analyses. An analysis of variance (ANOVA) was performed on the data set, with modality order, practice block, pattern, and pair type as the factors of particular interest. Computations of the two groups of subjects' perceptual sensitivity $\left(d^{\prime}\right)$ and response criteria $\left(B^{\prime \prime}\right)$ as a function of practice were also made. Multidimensional scaling (MDS) solutions were then derived from the confusion matrices.

\section{ANOVA of Discrimination Accuracy}

Figure 2 shows that mean accuracy (the probability with which subjects correctly determined sameness and difference) improved significantly with practice $[F(7,98)=$ $11.34, p<.001$ ]. Also evident in Figure 2 is the significant effect of pair type on accuracy: that is, in both groups, the subjects' mean probability of identifying a same pair correctly was greater than the mean probability of identifying a different pair correctly $[F(1,14)=23.29, p<$ $.001]$. For the visual-vibrotactile order group, the respective mean probabilities were 0.755 and 0.584 ; for the vibrotactile-visual order group, they were 0.655 and 0.550 . This interaction of modality order and pair type was not significant $[F(1,14)=1.36]$, nor was it altered significantly with practice $[F(7,98)<1]$.

Although the main effect of modality order was not significant $[F(1,14)=1.23]$, the modality order $\times$ practice block interaction was reliable $[F(7,98)=2.43, p<.02]$, indicating that as exposure to the patterns increased, the mean overall accuracy of the group that was exposed to the patterns first in the visual modality was improving more rapidly. After the first of the eight blocks of practice, both groups were performing at essentially chance levels (the visual-vibrotactile group $=0.478$ and the

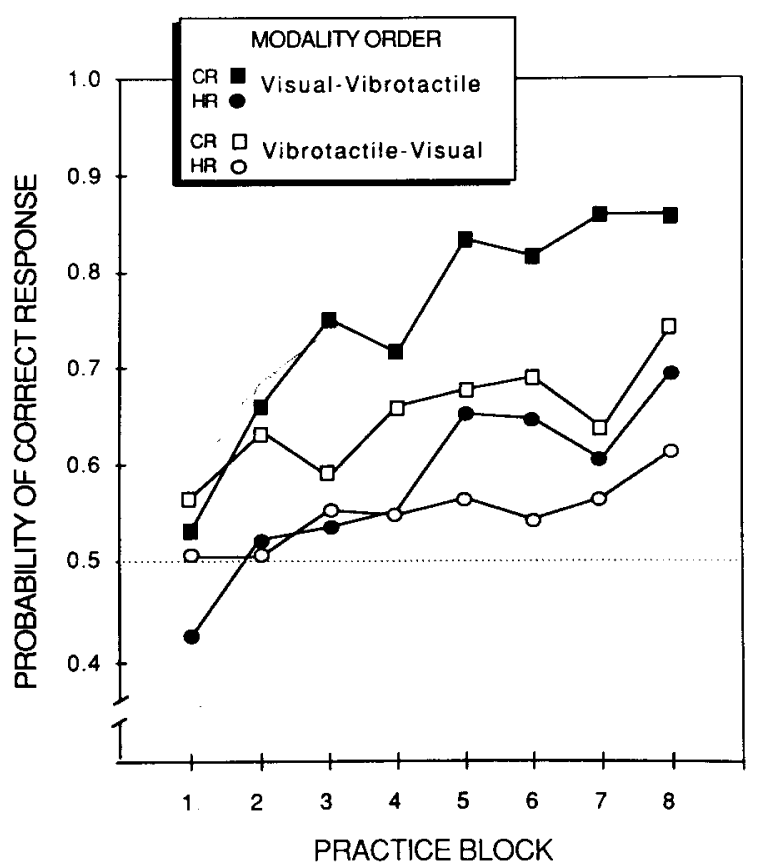

Figure 2. Mean probabilities of correct responses, for the two groups of subjects, as a function of pair type (same/different) and practice block. HR represents the probability of responding "different," given different shapes; CR represents the probability "same") same. The dotted line represents chance performance. 


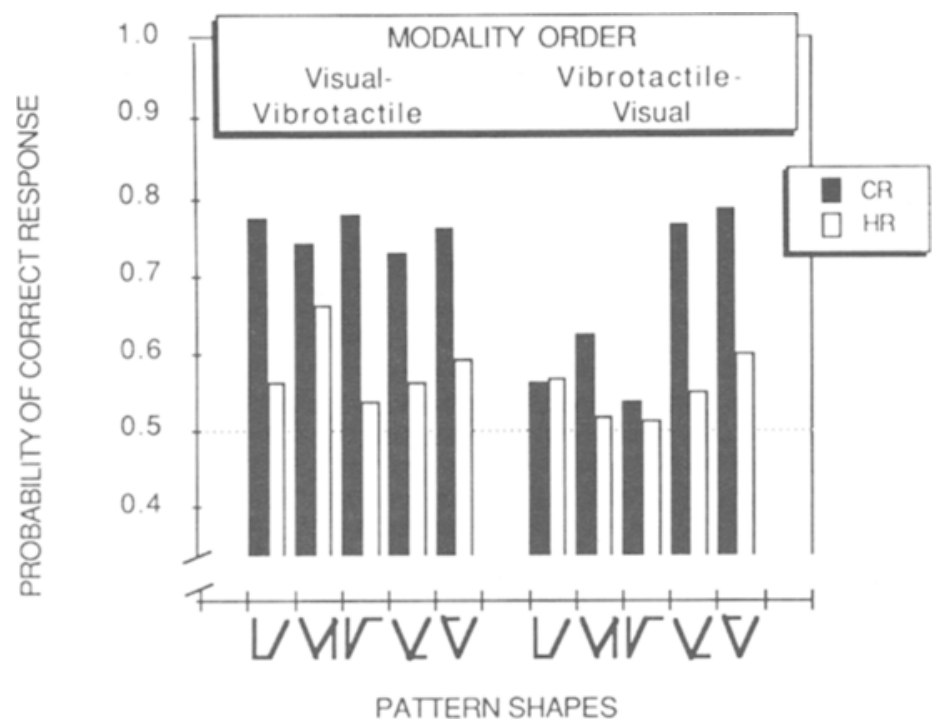

Figure 3. Mean probabilities of correct responses, for the two modality orders, as a function of pair type (same/different) and pattern shape. The shapes on the abscissa correspond to those shown in Figure 1.

vibrotactile-visual group $=.538$ ), but by the end of the eighth block they had improved to quite different levels $(0.781$ and 0.684 , respectively).

The main effect of pattern was also significant $[F(4,56)=$ $4.01, p<.007]$. Moreover, pattern interacted significantly with modality order $[F(4,56)=4.80, p<.003]$ and with modality order and pair type $[F(4,56)=5.15$, $p<.002]$. The latter interaction is shown in Figure 3 . What the figure reveals about the interaction is that (1) for both groups the probability of correctly discriminating different patterns was reasonably consistent (if low), and (2) for the visual-vibrotactile group, there was consistency in the probability of correctly judging same shapes; yet (3) this consistency is not evident for the accuracy with which same shapes were correctly judged by the subjects in the vibrotactile-visual group. That is, Patterns D and E (see Figure 1), when presented as same pairs vibrotactually rather than visually, were correctly judged to be identical with a probability equivalent to their presentation visually rather than vibrotactually $\left[p\left(\right.\right.$ same $\left./ \mathrm{D}_{\text {vib }}-\mathrm{D}_{\mathrm{vis}}\right)=0.766$, and $p\left(\right.$ same $\left./ \mathrm{E}_{\mathrm{vib}}-\mathrm{E}_{\mathrm{vis}}\right)=$ $0.785]$. The proportion of correct "same" responses for Patterns A, B, and C, conversely, were far lower; indeed, they are equivalent to their respective hit rates. No other higher order effects were found.

\section{Signal Detection Analysis}

For each of the two groups over practice blocks, estimations of $d^{\prime}$ and $B^{\prime \prime}$ were also computed. These two functions are shown in Figures 4 and 5, respectively. The $d^{\prime}$ estimates essentially recapitulate the modality order $\times$ practice block interaction described above; that is, although subjects in both groups show marginal sensitivity to the similarities in the structures during the initial practice blocks, they show considerable improvement with repeated exposure to the events. This improvement, however, is notably larger and more rapid for the visualvibrotactile group; the slopes of the best linear fits of the two functions shown in Figure 4 are 0.22 for the visualvibrotactile group and 0.09 for the vibrotactile-visual group.

$B^{\prime \prime}$ is a measure of the subject's response criterion and is similar to the more traditional measure, $\beta$ (Grier, 1971). Estimates of $B^{\prime \prime}$ can take any value between +1.00 and

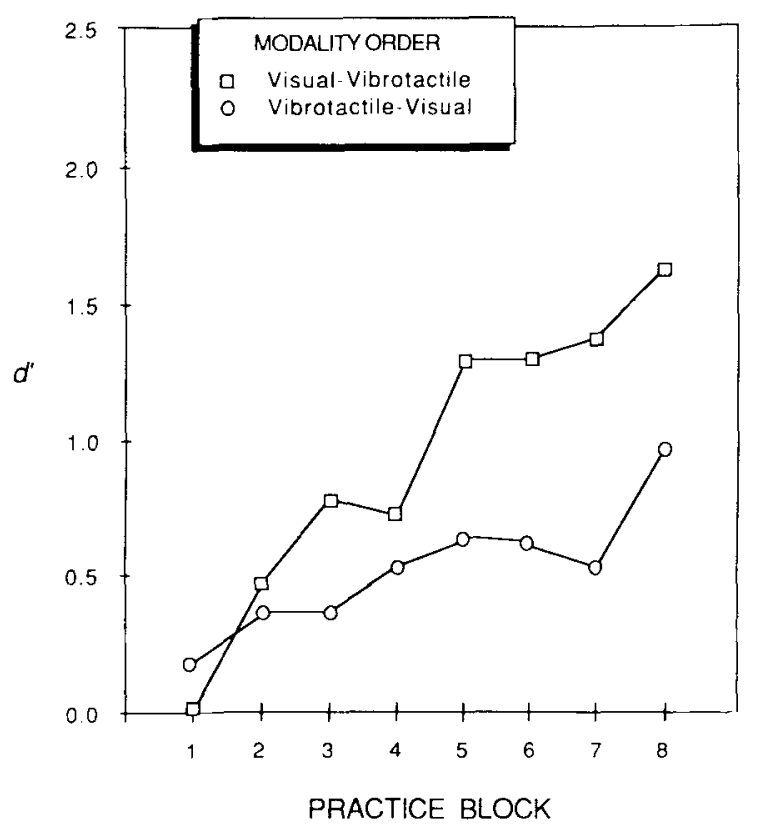

Figure 4. Mean sensitivity estimates $\left(d^{\prime}\right)$ for the two modality orders as a function of practice. 


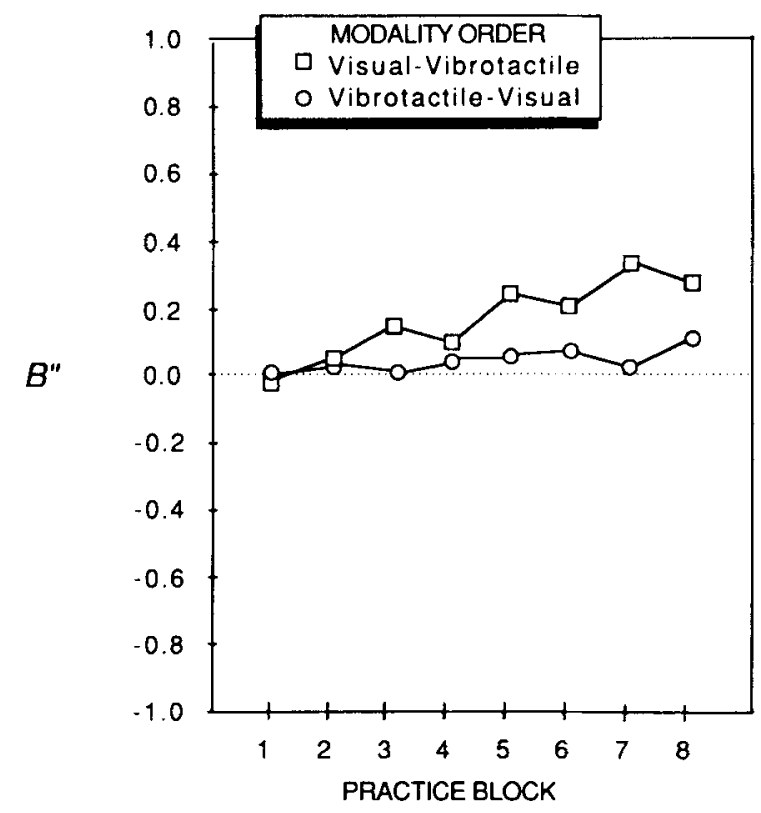

Figure 5. Mean response criterion estimates $\left(B^{n}\right)$ for the two modality orders as a function of practice.

-1.00 , with the positive sign indicating a tendency toward responding "same." As Figure 5 shows, the subjects in both groups revealed the use of a strict criterion of difference: Almost all points are positive, indicating that the subjects tended to be biased toward responding "same." However, this tendency was greater and increased more rapidly for the visual-vibrotactile group.

\section{Multidimensional Scaling}

An analysis was also performed on the data matrices containing the number of times subjects correctly recognized that the two patterns on any given trial were different. These $5 \times 5$ matrices were entered into an MDS analysis to produce a spatial representation of the degree of difference perceived between patterns. The closer any two patterns in the resultant space, the more difficult it was for subjects to differentiate the patterns. The dimensions of the spatial representation are those which account for the most variance in the data. MDS does not, however, provide an interpretation of these dimensions. The analysis presented here was conducted using the INDSCAL model of MDS (Carroll \& Chang, 1970; Carroll \& Wish, 1974), which allows for the analysis of more than one data matrix at a time. Unlike most MDS techniques, INDSCAL produces unique dimensions, ones that cannot be rotated or otherwise transformed without changing the solution in a fundamental way. Due to this property, Carroll and Wish (1974) suggest that INDSCAL dimensions correspond to basic perceptual processes whose salience may vary among individual subjects.

The analysis was conducted separately for the two groups of subjects. The overall purpose of the analysis was to determine whether the discriminability of patterns was qualitatively different for the two orders of presentation. A secondary purpose of the analysis was to evaluate the characteristics of the learning that was occurring across the blocks of the experiment. To do this, four data matrices (summed over subjects) were entered into the analysis, one for each consecutive pair of practice blocks (i.e., Blocks 1-2, 3-4, 5-6, and 7-8).

The separate MDS solutions for the two modality orders are presented in Figure 6 . The solutions provide a satisfying fit to the data, accounting for $83.5 \%$ of the variance in the visual-vibrotactile condition data and for $78.2 \%$ of the variance in the vibrotactile-visual condition data. (Using a single matrix for each condition and the Euclidean model, these percentages increase to $94.7 \%$ and $88.5 \%$, respectively.) Although the solutions for the two conditions are similar in some respects, there are also some obvious differences, and it is the consideration of the differences that seems particularly useful.

In our previous report (Epstein et al., 1989), we also derived two-dimensional solutions for eight patterns closely resembling those employed here. We tentatively concluded that for those patterns, spatial frequency and orientation

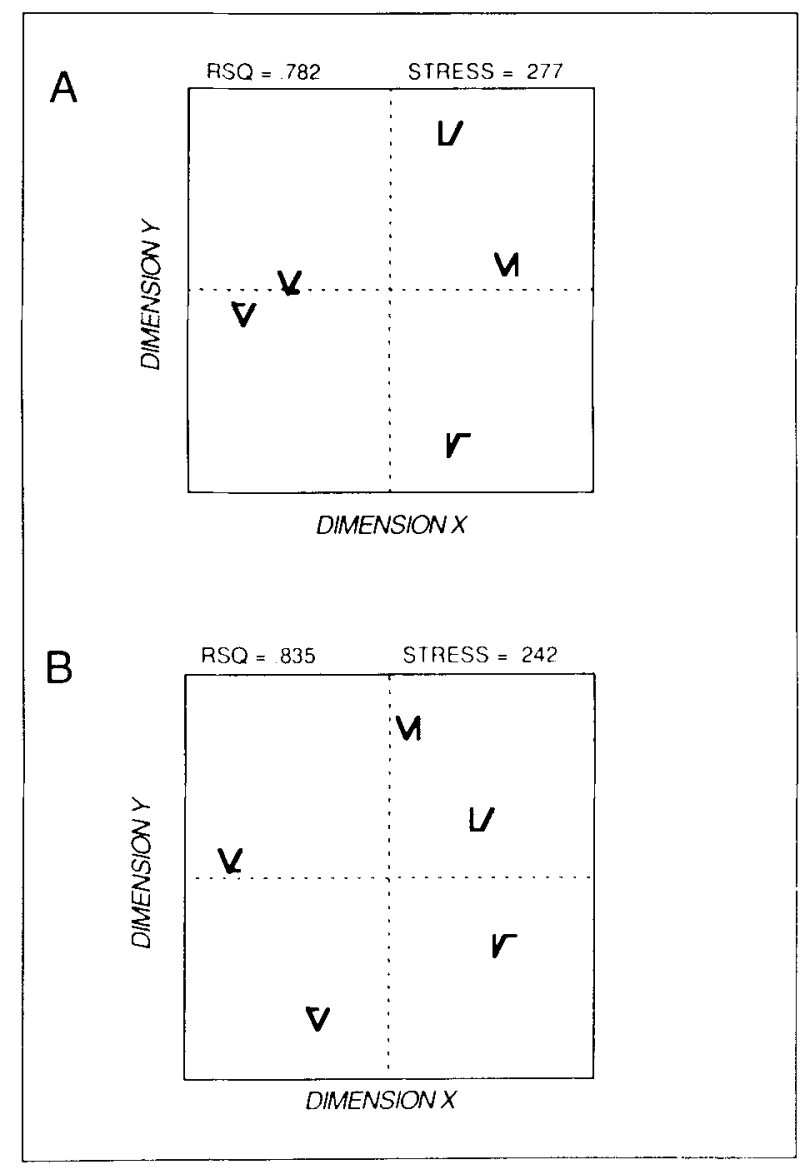

Figure 6. Resultant two-dimensional INDSCAL solutions: The upper panel (A) is the derived solution for the vibrotactile-visual group; the lower panel (B) is for the visual-vibrotactlle group. (RSQ = squared reliability coefmcient.) 
(i.e., whether the patterns had distinctive "openings" at the top or at the bottom) were the salient distinguishing features. By spatial frequency, we mean the intensity modulation resulting from a given pattern's component variably spaced line segments moving laterally across the array, rather than the technical Fourier representation. The resultant solution for the vibrotactile-visual group in this experiment (shown in the upper panel) suggests that the intensity distribution (with respect to the patterns' horizontal dimension, the direction of motion) is again a salient distinctive feature of the displays. The vertical axis of the solution (dimension $Y$ ) reveals that Pattern $\mathrm{A}$, which would be assigned the lowest spatial frequency, and Pattern C, with the highest, are maximally separated, while the remaining patterns have similar coordinates on the dimension. The latter patterns, it will be noted, all contain a V-form in their shapes. They differ, however, in the attached third line; Patterns D and E contain a horizontal line, but Pattern B contains an attached vertical line. It will be observed that in the solution, Patterns $\mathrm{D}$ and $\mathrm{E}$ share very similar coordinates and hence are highly confusable, an outcome that seems attributable to the fact that during lateral motion their horizontal line segments are present at the same time as the $\mathrm{V}$-shape, and may have been difficult to resolve spatially from that part of the pattern. In addition, as we noted in our previous report (Epstein et al., 1989), horizontal lines moving laterally create little of the spatiotemporal variation that seems critical for the recovery of shape in such tasks (Casco \& Morgan, 1984; Rock, 1981).

In the vibrotactile-visual group, dimension $X$ was of equivalent salience (as measured by the variance in the solution that it accounts for), but it is more difficult to provide with a single interpretive label. By our definition of orientation (see above), the patterns do not differ since they all have "openings" toward the top. This label cannot apply here. As is apparent, there is a conspicuous alignment of patterns, with $\mathrm{A}, \mathrm{B}$, and $\mathrm{C}$ on the right end of the axis and $D$ and $E$ on the left. It will also be recalled that Patterns D and E were both correctly judged to be same (when presented as same pairs) at an accuracy level comparable to that for the pattern pairs in the visualvibrotactile condition (see Figure 3 ). The relational variable that underlies this alignment is apparently complex and will require further assessment.

The resultant solution for the visual-vibrotactile group, shown in the lower panel, does not reveal a high degree of similarity in the alignment of the five patterns in either dimension. In this respect, we have additional confirmation of the nonequivalence of the perceptual representations associated with the two orders of presentation. Although relative perceptual similarity among certain pairs is evident in both solutions (see, e.g., A and B), the patterns can be seen to occupy quite unique coordinates. Unlike the previous solution, the visual-vibrotactile solution is arranged in an approximately circular configuration. Although the precise specification of dimensions is not possible here, the important finding is that the two solutions differ qualitatively. This suggests that pairwise perceptual similarities differ according to which modality is presented first with the dynamic shape in the discrimination task (i.e., whether a representation of the visual or vibrotactile shape serves as the standard for comparison).

In addition to evaluations of the overall MDS configurations, the INDSCAL analysis performed here allows an assessment of the degree to which subjects were relying on the MDS dimensions across blocks. If the subjects were learning over the course of the experiment, they should have been progressively improving in their ability to extract the dimensions of difference specifying pattern shape. Given that MDS techniques are designed to assess the extent to which systematic use of dimensions of difference is evident in a set of confusion data, it should be the case that the amount of variance in the confusion data accounted for by the MDS solution will increase as the ability to extract the information needed to accurately discriminate among the patterns improves. That is, if learning consists of the increased ability to extract diagnostic dimensions of difference among patterns, then one would expect that the amount of variance accounted for by an MDS solution would increase from earlier to later trial blocks.

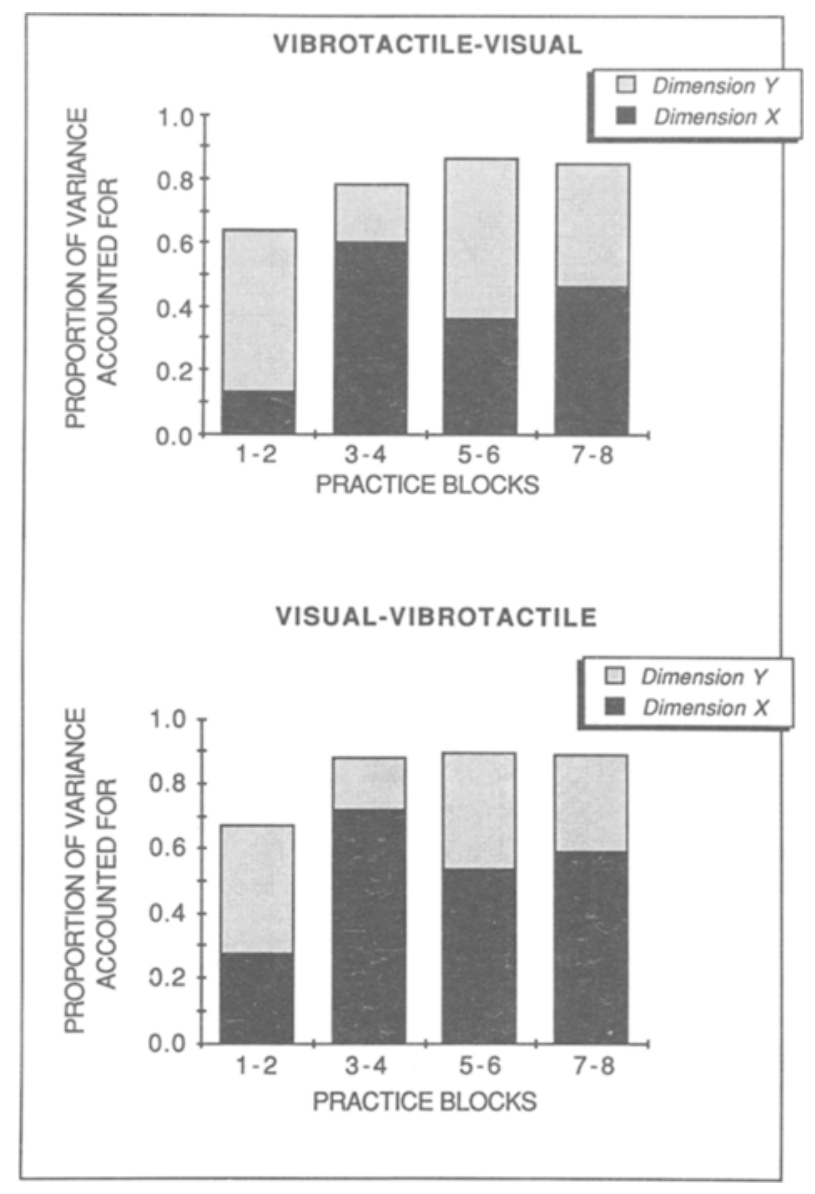

Figure 7. Estimates of the variance accounted for by each of the two dimensions of the INDSCAL solutions, for each of the two groups. 
The subjects' mean reliance on each of the MDS dimensions across blocks appears in Figure 7. Note first that for both groups there is a consistent overall improvement in the fit of the MDS solutions as one progresses from the early blocks to the later blocks. Especially for the visual-vibrotactile group, the variance accounted for by the MDS solution approaches asymptote in later blocks. A second finding is that the subjects' reliance on the $X$ dimension relative to the $Y$-dimension changes over time. Although initially the changes are somewhat unpredictable, in later blocks there appears to be a trend toward relying on both dimensions to approximately the same extent. Overall, this suggests that subjects are indeed learning to extract and use the diagnostic dimensions of difference among the patterns.

\section{DISCUSSION}

The results of the present experiment extend the generality of our (Epstein et al., 1989) findings by demonstrating perceptual learning in an intermodal same/different judgment task that called for comparison between dynamic patterns in both the visual and vibrotactile modalities. Two aspects of the data that appear to be of particular significance are the focus of the discussion.

The first concerns the main effect of pair type on subjects' performance. Although the correct rejection ("same"/ same) rate was significantly higher than the hit ("different"/ different) rate for both groups of subjects, both correct rejections and hits tended to increase at comparable rates from block to block. The superiority of correct rejections over hits seems to reflect a relatively strong bias to respond "same." In fact, signal detection analysis revealed that the response bias $\left(B^{\prime \prime}\right)$ favoring "same," as well as the perceptual sensitivity $\left(d^{\prime}\right)$ to characteristics of shape, were increasing regularly across blocks. The increase in both $B^{\prime \prime}$ and $d^{\prime}$ was especially great in the visualvibrotactile condition.

Although the decision criterion is technically construed to be independent of perceptual sensitivity, the similarity of the $B^{\prime \prime}$ and $d^{\prime}$ (see Figures 4 and 5) functions evidenced in this experiment is noteworthy. In this task, perceptual sensitivity is positively correlated with the stringency of the decision criterion regarding the degree of difference required in order to respond "different." One possible account of this correlation is that the decision criterion is continually reset relative to the degree or strength of the perceived difference between pairs of dynamic patterns rather than relative to the degree of similarity perceived between patterns.

Given that both same and different pairs of patterns produced different proximal events in terms of modality and direction of motion, relevant differences could include only those relational characteristics that contribute to the specification of pattern shape. The subjects must have been improving both in their selective extraction of differences diagnostic of pattern shape and in their ability to discriminate along the relevant dimensions. As percep- tual sensitivity increased in this way, these diagnostic dimensions could more easily be accessed to serve as the anchor for establishing the decision criterion.

This characterization of the relationship between perceptual sensitivity and the decision criterion appears consistent with the intent of $\mathrm{E}$. J. Gibson's differentiation theory (see, e.g., E. J. Gibson, 1969; E. J. Gibson \& J. J. Gibson, 1955). Gibson claims that increases in perceptual sensitivity are based on increases in the ability to extract the diagnostic dimensions of difference among patterns. The extraction of these differences may serve to focus the perceiver's attention on other relational variables specifying differences in pattern structure, and could contribute to the setting of a decision criterion.

A novel finding emerged in this experiment in the form of the event modality order $\times$ practice interaction. For the first two blocks of trials, performance was comparable under the two modality conditions; starting with the third block of trials, however, performance under the two conditions diverged substantially. Performance under the visualvibrotactile condition improved with practice significantly more rapidly than performance under the vibrotactilevisual condition. (Related outcomes are by no means unusual in the literature; Jones [1981, pp. 120-121] has summarized the results of 30 studies of visual-haptic shape matching; in the majority of cases, visual-haptic performance was superior to haptic-visual performance, although in a substantial minority of instances, no differences in performance were observed.)

An interpretation of the interaction may be developed within the framework of the differentiation theory of perceptual learning. According to this formulation, practice affords the opportunity for the education of attention leading to the detection of the spatiotemporal structures of stimulation that distinguish the patterns. It is plausible to assume that other variables that contribute to the focusing of attention will also contribute to improvements in performance. Exposure to a visual display preceding presentation of the vibrotactile display may serve this purpose. Very early in practice, patterns presented visually are perceptually differentiated and identified. When an articulated visual representation is made available first, this representation may act to direct attention to the isomorphic vibrotactile structures that differentiate the vibrotactile patterns. The resulting effect will be enhanced performance. In the case of the vibrotactile-visual condition, the visual representation cannot act retroactively to enhance the detection of the informative vibrotactile structures. When the vibrotactile patterns are presented first, improvement in their differentiation will depend entirely on the education of attention that is associated with repeated, motivated encounters. One consequence of the argument that performance in the visual-vibrotactile condition benefits from both practice and the directive influence of articulated visual representation, whereas performance in the vibrotactile-visual condition benefits only from practice, is that performance under the former condition improves more rapidly. 
The obtained interaction and our interpretation of it may appear to be at odds with the notion that amodal information underlies intermodal comparisons. If the information is amodal-that is, if the information is available in the same form in both modalities-it may seem that performance should be indifferent to the order of modality. The resolution depends on distinguishing between the availability and the accessibility of information. Although information in the form of isomorphic spatiotemporal structures is equally available in both conditions, we are arguing that the information is more accessible in the visual modality, and that it is the combined difference in the accessibility and the temporal order in which this information is accessed that underlies the interaction.

The application of this distinction to our data may be clarified by reference to a model of intermodal (visualtactile) pattern perception that has been developed by Loomis (e.g., Loomis, 1980, 1981, 1982; Loomis \& Lederman, 1986). Central to this model is the claim that in both perceptual systems, spatial and temporal sensitivity (separately or interactively) "place quantifiable limits on perceptual capacity" (Loomis \& Lederman, 1986, p. 31.4). In the case of tactile perception, these limits are substantially more severe (e.g., spatial resolution and acuity are lower). From the evidence Loomis $(1981$, p. 7$)$ has modeled tactile pattern perception via the incorporation of a (functional) low band-pass spatial filter that acts on the stimulation, serving to partially or totally attenuate the information contained in it. As a consequence, the perceptual representation of a tactile shape is a degraded version of the proximal pattern of stimulation (relative to the precision with which a visual event would be represented). For Loomis (1981), the primary factors serving to limit tactile spatial resolution are located in the physiological, mechanoreceptive properties of the skin (i.e., in its static and dynamic mechanical properties, and in receptor field densities, neural pathways, and cortical organization).

One might presume from Loomis's model that tactile perception is constrained by the nonavailability of certain information to the perceptual system (although, of course, we are not necessarily attributing this interpretation to Loomis). However, we are arguing that Loomis's model and the data supporting it refer to the relative accessibility of the information, not to the availability of information. Information for the recovery of the pattern shapes, we are suggesting, either exists or it does not. Accessibility, on the other hand, is more a matter of degree, and it may be enhanced or reduced according to many different context-dependent factors. Indeed there may be conditions of perceptual experience where tactile accessibility is greater than visual accessibility (such as in the case of surface texture perception; see Loomis \& Lederman, 1986) and where one might observe the opposite effects of modality order on perceptual differentiation and learning. Increased accessibility to information is made manifest by, among other things, perceptual learning. Our claim is that when the first pattern was visually received, not only was more information about its shape accessible, but this access (by means that are yet to be precisely specified) served to enhance the accessibility of the information that specified pattern shape in the subsequent vibrotactile event.

\section{REFERENCES}

Anstis, S. M., \& AtKinson, J. (1967). Distortions in moving figures viewed through a stationary slit. American Journal of Psychology, 80, 572-585.

Carroll, J. D., \& Chang, J. (1970). Analysis of individual differences in multidimensional scaling via an $N$-way generalization of "Eckart-Young" decomposition. Psychometrika, 35, 283-319.

Carroll, J. D., Wish, M. (1974). Models and methods for threeway multidimensional scaling. In D. H. Krantz, R. C. Atkinson, R. D. Luce, \& P. Suppes (Eds.), Contemporary developments in mathematical psychology: Vol. 2. Measurement, psychophysics, and neural information processing (pp. 75-123). San Francisco: W. H. Freeman.

Casco, C., \& Morgan, M. J. (1984). The relationship between space and time in the perception of stimuli moving behind a slit. Perception, 13, 429-441.

EPSTEIN, W. (1985). Transmodal perception and amodal information. In D. Warren \& E. Strelow (Eds.), Electronic spatial sensing for the blind. Dordrecht: Martinus Nijhoff.

Epstein, W., Hughes, B., Schneider, S., \& BaCh-y-Rita, P. (1989). Perceptual learning of spatiotemporal events: Evidence from an unfamiliar modality. Journal of Experimental Psychology: Human Perception \& Performance, 15, 28-44.

GaRBIN, C. P. (1988). Visual-haptic perceptual nonequivalence for shape information and its impact upon cross-modal performance. Journal of Experimental Psychology: Human Perception \& Performance, 14, 547-553.

Gibson, E. J. (1969). Principles of perceptual learning and development. New York: Appleton-Century-Crofts.

Gibson, E. J., \& Gibson, J. J. (1955). Perceptual learning: Differentiation or enrichment? Psychological Review, 62, 32-41.

Gisson, 1. J. (1966). The senses considered as perceptual systems. Boston: Houghton Mifflin.

GiBson, J. J. (1979). The ecological approach to visual perception. Boston: Houghton Mifflin.

GriER, J. B. (1971). Nonparametric indexes for sensitivity and bias: Computing formulas. Psychological Bulletin, 75, 424-429

JONES, B. (1981). The developmental significance of cross-modal matching. In R. Walk \& H. L. Pick (Eds.), Intersensory perceprion and sensory integration (pp. 128-148). New York: Plenum.

Loomis, J. M. (1980). Interaction of display mode and character size in vibrotactile letter recognition. Bulletin of the Psychonomic Society, 16, 385-387.

Loomis, J. M. (1981). Tactile pattem perception. Perception, 10, 5-27.

LoOMIs, J. M. (1982). Analysis of tactile and visual confusion matrices Perception \& Psychophysics, 31, 41-52.

Loomis, 1. M., Lederman, S. J. (1986). Tactual perception. In K. Boff, L. Kaufman, \& J. Thomas (Eds.), Handbook of perception and human performance (Vol. 2, pp. 31 1-31.41). New York: Wiley.

MARKS, L. E. (1978). The unity of the senses: Interrelations among the modalities. New York: Academic Press.

Morgan, M. J., Findlay, J. M., \& Watt, R. J. (1982). Aperture viewing: A review and synthesis. Quarterly Journal of Experimental Psychology, 34A, 211-233.

Rock, I. (1981). Anorthoscopic perception. Scientific American, 244 $145-153$.

Schneider, S. L., Hughes, B., Epstein, W., \& Bach-y-Rita, P. (1986). The detection of length and orientation changes in dynamic vibrotactile patterns. Perception \& Psychophysics, 40, 290-300.

(Manuscript received August 7, 1989; revision accepted for publication February $7,1990$. 